\title{
A Solution for Identification of Bird's Nests on Transmission Lines with UAV Patrol
}

\author{
Qinghua Wang \\ Fuzhou Power Supply Company of State Grid Fujian Electric Power Company, Fuzhou 350009, \\ China. \\ wangqinghua9978@163.com
}

\begin{abstract}
Birds often disturb the normal operation of transmission lines during their activities in the nature. Now, it is common to rely on workers that check, identify and remove bird's nests on transmission lines, but their observation is less efficient and geographically limited. This paper proposes a solution for identification of bird's nests on transmission lines based on feature identification, which locates the pole tower of transmission line by LSD line detection, Harris corner detection and morphological closing operation, and detects the bird's nests within the range of pole tower based on their shape and color features, in order to identify the bird's nests accurately.
\end{abstract}

Keywords: Lerial image; transmission lines; identification of bird's nests; feature identification.

\section{Introduction}

Transmission lines play a very important role in the electric power system by bringing the electricity to every household, so their massive failure will cause immeasurable losses to national economy. The electric power utility should therefore pay much attention to the safety of transmission lines.

To guarantee safe and reliable operation of power grids, it is necessary to monitor, make early warning on and prevent bird's nesting on transmission line pole towers. Now, transmission lines rely on patrol for visual check with the assistance of observation and detection instruments, which covers the bird's nests on pole tower, and the operation status of grounding wire and its fittings, insulators and other components. However, visual observation is less efficient and geographically limited, so workers have undertaken plenty of tedious work to identify the information for early warning but still less efficiently.

Traditional patrol faces large workload and harsh conditions, especially for transmission lines in mountainous areas and across big rivers, during freezing weather, flood, earthquake, and landslide or at night, which is time-consuming, costly, difficult and risky. In recent years, unmanned aerial vehicle (UAV) appears as a maturating technology to achieve surface observation, geological exploration and line patrol by means of low altitude remote sensing. Especially, UAV patrol has gradually become an important focus of development in the patrol check of high-voltage transmission lines thanks to its efficiency, accuracy, and safety, etc. A great number of aerial images gained from aerial patrol are processed and analyzed to identify the failures and defects of transmission lines and perform the monitoring and early warning of damages to electric facilities in special geological environment.

However, aerial patrol gathers a large number of images or videos, so it must be very tedious to directly process them by artificial screening, analysis and judgment thereafter, which may also easily miss key information. If workers are arranged to monitor, judge and identify the abnormalities of transmission lines in a real-time manner, it is more arduous to process and easier to omit key information or make false alarms. If intelligent identification is employed to identify and analyze the potential threats to bird's nests on transmission line pole towers, the quality of images collected becomes the key.

The images collected from aerial patrol experience degradation to difference degrees. Images degrade for many reasons, e.g. image distortion caused by aberration, diffraction and limited bandwidth of optical system; remote sensing image distortion due to solar radiation, cloud covering and perturbation effect of atmospheric turbulence; and image geometrical distortion attributed to 
shooting stance of imaging device and nonlinearity of optical-electrical converter. Among them, motion blur and noises have very significance influence. Additionally, the changing natural environment and landform of transmission corridor due to change of seasons will complicate the background of image collection, lower the contrast and increase the disturbances, while the complication of image background is further worsened by other natural landscapes and artificial buildings. Under the complicated natural background, the extraction and identification of target images is another technical bottleneck in the automatic diagnosis of key equipment for transmission lines.

Therefore, computer visual analysis technology in a sense of artificial intelligence is introduced to add the features of eye identification and brain analysis to the existing patrol system. Through visual analysis, the potential threats including bird's nests on transmission line pole towers are identified and analyzed to realize the real-time warning and lower the accident rate.

\section{Algorithm for Detection of Bird's Nests on Transmission Lines Based on Feature Identification}

For diverse shooting angles of images taken by UAV during patrol, and different environmental influence of light and climate during shooting, a series of complicated operations must be conducted for images considering the complicated field background of transmission lines. This algorithm mainly identifies transmission line pole towers and then bird's nests based on shape and color features by judging whether it is a bird's nest on the pole tower or similar image in the background. The specific process is as follows in figure 1:

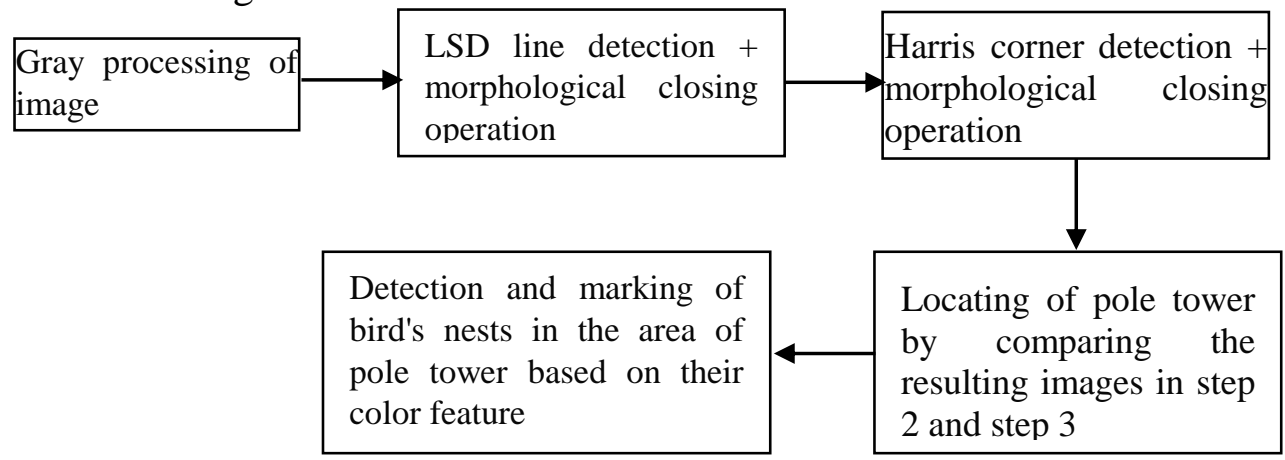

Fig 1. Algorithm flowchart

\subsection{Gray processing of images}

UAV patrol collects color images in RGB color model. Images are processed for three components of RGB respectively, but RGB cannot reflect the morphological features of images practically, and color mixing is only carried out in the optical principles.

Now, there are many other color models, e.g. HIS model, which uses three components, i.e. hue, saturation and intensity to represent different colors. HIS fits better the visual features of human being than RGB.

The color of each pixel in color image depends on three components, i.e. R, G and B, each of which ranges from 0 to 255 , giving more than 16 million $\left(255^{*} 255^{* 255)}\right.$ kinds of color to each pixel. Differently, gray image is a special image with the equal value for three components, $\mathrm{R}, \mathrm{G}$ and $\mathrm{B}$, so each pixel has 255 possible kinds of color. In digital image processing, images in different formats are converted into gray images to simplify the subsequent computation for images. Like color image, gray image can still reflect the distribution and features of global and local color and brightness.

In the color space of YUV, the physical significance of the component Y is the brightness of point, which reflects the level of brightness. Based on the relationship of variation between RGB and YUV color space, the correspondence of brightness $Y$ to three color components $R, G$ and $B$ can be established as follows: $\mathrm{Y}=0.3 \mathrm{R}+0.59 \mathrm{G}+0.11 \mathrm{~B}$. This value of brightness can be used to represent the gray value of image. 


\subsection{LSD line detection}

LSD line detection aims to detect the local straight outline of image. LSD algorithm should first generate a series of line segments based on the previous gray scale chart obtained the image processing.

The first step of LSD algorithm is to shrink the input image to $80 \%$ of its original size, with an aim to alleviate and even eliminate the aliasing effect in many images. Then, gradient computation is carried out to obtain the gray value of each pixel point on the gray scale chart as follows:

\begin{tabular}{c|c|c|c}
$\ddots$ & $\vdots$ & $\vdots$ & $\cdots$ \\
\hline$\cdots$ & $i(x, y)$ & $i(x+1, y)$ & $\cdots$ \\
\hline$\cdots$ & $i(x, y+1)$ & $i(x+1, y+1)$ & $\cdots$ \\
\hline$\cdots$ & $\vdots$ & $\vdots$ & $\ddots$
\end{tabular}

Hence, the gradient computation of image employs the following formula:

$$
\begin{aligned}
& g_{x}(x, y)=\frac{i(x+1, y)+i(x+1, y+1)-i(x, y)-i(x, y+1)}{2} \\
& g_{y}(x, y)=\frac{i(x+1, y)+i(x+1, y+1)-i(x, y)-i(x, y+1)}{2}
\end{aligned}
$$

The gradient amplitude is calculated with the following formula:

$$
G_{(x, y)}=\sqrt{g_{x}^{2}(x+y)+g_{y}^{2}(x, y)}
$$

Subsequently, the calculated gradients are sequenced to obtain 1024 arrays with even interval between 0 and maximum gradient amplitude. The pixels are classified into these 1024 arrays based on the corresponding gradient amplitude. The pixel with the maximum gradient is taken as the seed point for sequencing.

The variation of gradient amplitude is shown in the sequence of calculated gradients. In other words, any gradient amplitude of small variation falls into the platform area or gentle area. The quantification of pixel may cause any error in the gradient computation, so it is necessary to set a gradient threshold, and any point under the threshold is overlooked.

In the sequence list, an unused pixel is taken as the seed point for recursion to expand the area. At last, rectangular estimation is conducted to finish the line detection with LSD algorithm.

\subsection{Harris corner detection}

First of all, a small local area is selected. If the window of this feature moves upwards in any direction, and the gray scale of the area in the window changes dramatically, there will be a corner in the window. If the window moves in any direction of this image, and the gray scale of image in the window does not change, there is not any corner in the window. If the window moves in a direction, and the gray scale of image in the window changes dramatically, but does not change in some other directions, the image in the window may be a length of line.

The process of Harris corner algorithm is to calculate the gradients of the image in two directions $\mathrm{X}$ and $\mathrm{Y}$, and then obtain the product of its gradients in such two directions. Gauss function is employed to weight the product into the element of matrix.

Harris value of each pixel is calculated, and any value larger than the threshold is overlooked.

The maximum suppression is made in the neighboring domain, while the local maximum is the corner in the image.

\subsection{Morphological Closing Operation}

Morphological closing operation is employed to eliminate small objects, separate objects at any slim point, and borders of large smooth objects without changing their area significantly.

Morphological erosion option is a process of eliminating the border points and make borders move inwards, so as to eliminate some small and meaningless objects.

Erosion algorithm:

The $3 \times 3$ structural element is employed to scan every pixel of image. The "AND" operation is conducted with structural element for binary image it covers. If the results are always 1, the pixel of the resulting image is 1 . Otherwise, it is 0 . Then, the binary image can be reduced by one round. 
Expansion is a process of combine all background points in contact with any object into the object, so as to expand the borders outwards. It can be used to fill the cavity in any object.

Expansion algorithm:

The $3 \times 3$ structural element is employed to scan every pixel of image. The "AND" operation is conducted with structural element for binary image it covers. If the results are always 0 , the pixel of the resulting image is 0 . Otherwise, it is 1 . Then, the binary image can be expanded by one round.

An image is processed with expansion algorithm followed by erosion algorithm to complete the morphological closing operation.

\section{Experiment Results and Analysis}

This paper classifies samples into training samples and test samples. The training samples contain 80 samples with bird's nest and 80 samples without bird's nest, while the test samples have 40 samples with bird's nest and 40 samples without bird's nest to be tested.

Table 1. The results number of samples

\begin{tabular}{|c|c|c|c|c|}
\hline \multirow{2}{*}{ Category } & \multicolumn{2}{|c|}{ Training Sample } & \multicolumn{2}{c|}{ Test Sample } \\
\cline { 2 - 5 } & With Bird's Nest & Without Bird's Nest & With Bird's Nest & Without Bird's Nest \\
\hline Sample Size & 80 & 80 & 40 & 40 \\
\hline Total & \multicolumn{2}{|c|}{160} & \multicolumn{2}{|c|}{80} \\
\hline
\end{tabular}

In the aerial images, there is complicated background for transmission lines. Among the videos or single images collected, there may be bird's nests at many places. For the image of pole tower under the complicated background, this paper effectively extracts the structural features of pole tower based on the clear structural information in the shape of pole tower, so as to guarantee the accuracy, efficiency and low spatial complexity of detection method for electric pole tower under the complicated background. Subsequently, bird's nests in the pole tower area are identified based on their shape and color so as to guarantee accurate identification. Hence, the algorithm proposed in this paper can correctly identify the bird's nests on the pole tower without being affected by other bird's nests in the background.

From these images taken in aerial patrol, clear images should be obtained as much as possible to provide the image of bird's nest on transmission line pole tower from different angles and in different scenarios. After these sample images are processed, the results of identification are shown in the following figure. In this process, bird's nests on transmission lines are identified correctly and marked.

\section{Conclusion}

Bird's nesting behavior affects the safe operation of transmission line pole towers, which leads to the short circuit or tripping of lines very easily. To guarantee the normal operation of power grid in the future, it is necessary to monitor, make early warning on and prevent bird's nesting on transmission lines. This paper proposes a solution for identification of bird's nests on transmission lines based on feature identification to accurately detect the bird's nests on the pole towers in the aerial images. In this solution, the pole tower of transmission line is located by LSD line detection, Harris corner detection and morphological closing operation, and then the bird's nests within the range of pole tower are detected based on their shape and color features, in order to identify the bird's nests accurately. However, this paper employs a limited number of samples. The study and analysis will be more comprehensive if enough samples are employed. 


\section{References}

[1] Wang Zhen. The Recognition of Bird's Nest on Transmission Line Towers Based on Video Image Processing Technology. Thesis (master). North China Electric Power University, 2013.

[2] Duan Wangwang, Tang Peng, Jin Weidong and Wei Pu. Detection of bird's nest on railway contact system based on HOG characteristics of key zones. Chinese Railways, 2015, (8): 73-76.

[3] Li Zhongning and Chen Fengxiang. UAV inspection application in transmission lines. Guizhou Electric Power Technology, 2012, 15(4): 83-86.

[4] Liu Gao and Zeng Yihui. Embedded video and technology of image recognition in the intelligent monitoring of overhead transmission lines. Computer Systems \& Applications, 2013, 22(11), 58-62.

[5] Tong Weiguo. Research on Power Line Identification and Status Detection Based on Aerial Image. Thesis (PhD). North China Electric Power University, 2011. 